http://dx.doi.org/10.35381/s.v.v4i8.1002

\title{
La influencia de los medios de comunicación en el envejecimiento activo
}

The influence of the media on active aging

Victoria Noemi Gutiérrez-Martínez

vgutierrez0282@uta.edu.ec

Postgrado de Medicina Familiar y Comunitaria, Facultad de Ciencias de la Salud, Universidad Técnica de Ambato, Ambato

Ecuador

https://orcid.org/0000-0003-2130-5463

Patricio Rodrigo-Maldonado

r.maldonado@uta.edu.ec

Postgrado de Medicina Familiar y Comunitaria, Facultad de Ciencias de la Salud, Universidad Técnica de Ambato, Ambato

Ecuador

https://orcid.org/0000-0002-4896-1586

Mayra Alexandra Vela-Chasiluisa mvela7463@uta.edu.ec

Postgrado de Medicina Familiar y Comunitaria, Facultad de Ciencias de la Salud, Universidad Técnica de Ambato, Ambato

Ecuador

https://orcid.org/0000-0002-0140-7246

Recepción: 15 de junio 2020

Revisado: 28 de julio 2020

Aprobación: 25 de agosto 2020

Publicación: 03 de septiembre 2020 


\title{
RESUMEN
}

El envejecimiento es un proceso continuo en el desarrollo del ser humano que es físico, biológico y social que se construye cada día para lograr las capacidades de cada persona. Objetivo: Determinar los factores asociados al envejecimiento activo, en especial las redes sociales, en una población de la sierra ecuatoriana. Metodología: Investigación de enfoque mixto de campo y de corte transversal, en 30 adultos mayores de la Parroquia Antonio José Holguín. Resultados: Se evidencia el bienestar físico el $90 \%$ de los participantes son independientes en actividades básicas de la vida diaria, y 63\% necesitan cierta ayuda en las actividades instrumentales. Conclusión: Las redes sociales influyen en el envejecimiento activo de las personas de la tercera edad. Existe una relación del bienestar físico, mental y social con el uso de redes sociales como medios de comunicación.

Descriptores: Salud pública; envejecimiento; dinámica poblacional. (Fuente DeCS, 2020).

\begin{abstract}
Aging is a continuous process in the development of the human being that is physical, biological and social that is built every day to achieve the capabilities of each person. Objective: To determine the factors associated with active aging, especially social networks, in a population of the Ecuadorian highlands. Methodology: Research with a mixed field and cross-sectional approach, in 30 elderly adults of the Antonio José Holguín Parish. Results: Physical well-being is evident in $90 \%$ of the participants who are independent in basic activities of daily living, and $63 \%$ need some help in instrumental activities. Conclusion: Social networks influence active aging in the elderly. There is a relationship between physical, mental, and social well-being and the use of social networks as a means of communication.
\end{abstract}

Descriptors: Public health; aging; population dynamics. (Source DeCS, 2020). 


\section{INTRODUCCIÓN}

El envejecimiento es un conjunto de modificaciones morfológicas y fisiológicas que van desde el momento del nacimiento, se ven modificado por la herencia, el ambiente y la conducta ${ }^{1}$. La salud, el bienestar y la productividad en la vejez dependen del entorno físico, entorno social, asuntos económicos y aspectos conductuales ${ }^{2}$.

A nivel mundial existe un rápido crecimiento de las personas mayores de 60 años, gracias a los avances en la higiene, suministro de agua y control de enfermedades infecciosas han reducido en el mundo. La dinámica familiar varía cuando existen personas adultas mayores, las cuales suelen vivir en soledad, bien sea porque han tomado esa decisión o porque su familia tiene roles sociales que no le permiten atenderlos permanentemente ${ }^{3}$. A nivel de Ecuador es un proceso transición demográfica de manera tardía para países desarrollados. En el año 2017 el porcentaje de personas de 65 años fue del 7,04\%. Para el 2025 superaría el $10 \%$ de la población total ${ }^{4}$. Las poblaciones enfrentan un aumento de personas envejecidas, con incremento en el uso de la tecnología digital que permite el envejecimiento activo ${ }^{5}$. El uso de la tecnología de información y comunicación es usado para conexión con familiares, amigos y búsqueda de actividades de ocio ${ }^{6}$.

${ }^{7}$ el uso de redes sociales es parte de la vida de los adultos mayores lo provoca que se incorporen a través de la tecnología de la comunicación para participar en actividades sociales. La red social más utilizada Facebook que provoca en las personas de la tercera edad reducir el aislamiento social y con participación activa ${ }^{8}$. El apoyo social y conexión social que es favorable para un bienestar positivo, con cambios en la autoeficacia percibida ${ }^{9}$.

El objetivo de esta investigación es determinar los factores asociados al envejecimiento activo, en especial las redes sociales, en una población de la sierra ecuatoriana. 


\section{METODOLOGÍA}

\section{Tipo y diseño de investigación}

Es una investigación de corte observacional transversal descriptivo.

\section{Muestra poblacional}

Se contó con 30 adultos mayores, de los cuales son 14 masculinos y 16 mujeres.

\section{Recopilación de información}

Se aplicó escala geriátrica integral del ministerio de salud pública del Ecuador y entrevista semiestructurada diseñada por los investigadores a partir de su observación en el proceso investigativo.

\section{Análisis de la información}

La escala geriátrica integral se analizó utilizándose el programa EViews 10 y la regresión lineal.

A partir de la entrevista semiestructurada se generaron categorías como: características sociodemográficas, bienestar físico, bienestar mental, bienestar social, factores asociados en especial redes sociales; aplicándose análisis de contenido para tal fin. 


\section{RESULTADOS}

Posteriormente de codificar y analizar la escala geriátrica integral y las entrevistas semiestructuradas, surgieron tres categorías.

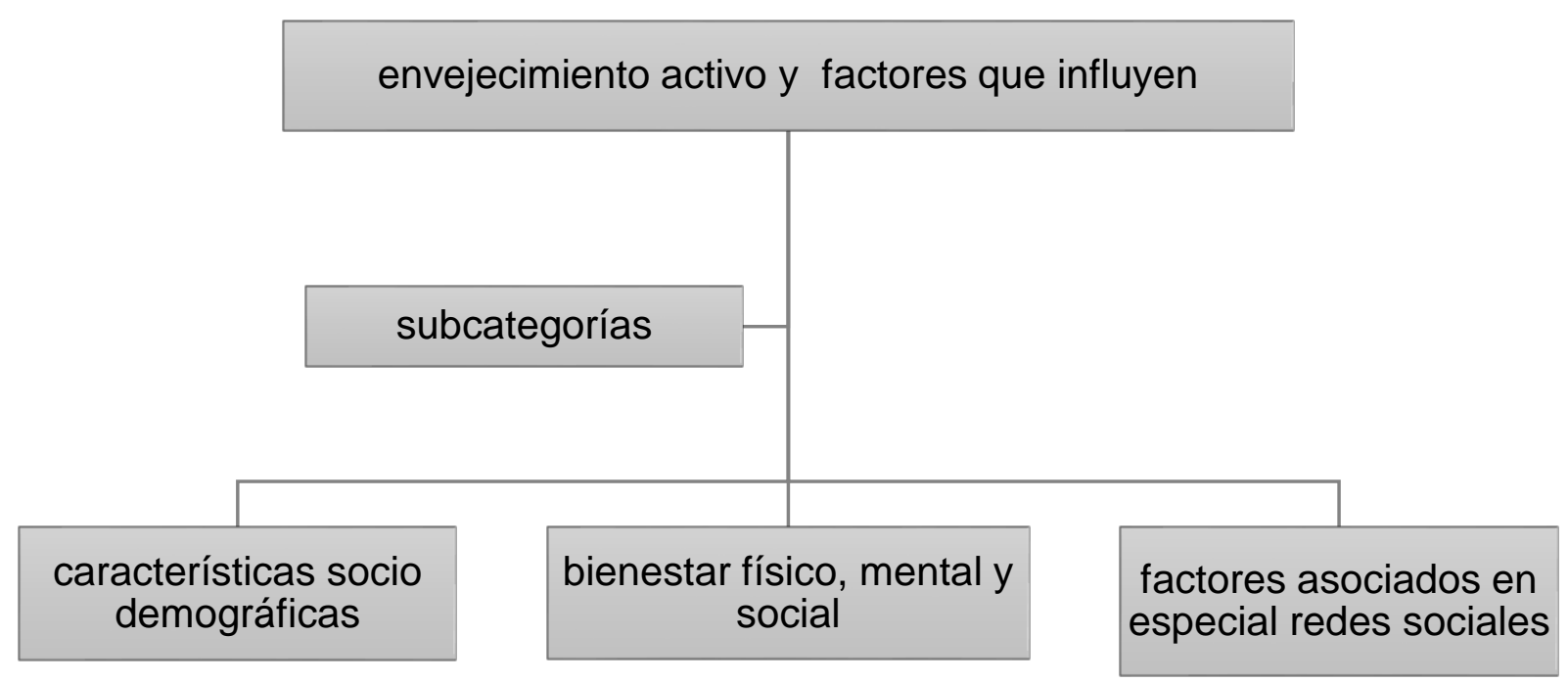

Figura 1. Escala geriátrica integral.

Fuente: Elaboración propia.

En la tabla 1 se representa las variables sociodemográficas en donde se incluye edad, sexo, estado civil y convivencia de un grupo de adultos mayores activos. Los porcentajes de las personas en un grupo de 65-74 años que corresponde al $50 \%$, con un predominio femenino con el 46,69\%. En cuanto al estado civil en el sexo masculino casados en un $33,3 \%$ y el $16,7 \%$ de femenino se encuentran viudas. 
Tabla 1.

Distribución de adultos mayores según grupo de edad y sexo, estado civil de la parroquia Antonio José Holguín en el 2019-2020.

\begin{tabular}{|c|c|c|c|c|c|c|}
\hline Grupo de edad & \multicolumn{2}{|c|}{ Masculino } & \multicolumn{2}{|c|}{ Femenino } & \multicolumn{2}{c|}{ Total } \\
\hline & $\mathrm{n}$ & $\%$ & $\mathrm{n}$ & $\%$ & $\mathrm{n}$ & $\%$ \\
\hline $\mathbf{6 5 - 7 4}$ & 8 & 26,70 & 7 & 23,33 & 15 & 50 \\
\hline $\mathbf{7 5 - 8 4}$ & 4 & 13,33 & 7 & 23,33 & 11 & 36,6 \\
\hline $\mathbf{8 5 - 9 4}$ & 1 & 3,33 & 1 & 3,33 & 2 & 6,7 \\
\hline $\mathbf{9 5}$ o más & 1 & 3,33 & 1 & 3,33 & 2 & 6,7 \\
\hline Total & 14 & 46,69 & 16 & 53,33 & 30 & 100 \\
\hline
\end{tabular}

\begin{tabular}{|c|c|c|c|c|c|c|}
\hline Estado civil & \multicolumn{2}{|c|}{ Masculino } & \multicolumn{2}{|c|}{ Femenino } & \multicolumn{2}{|c|}{ total } \\
\hline & $\mathrm{n}$ & $\%$ & $\mathrm{n}$ & $\%$ & $\mathrm{n}$ & $\%$ \\
\hline soltero & 0 & 0 & 3 & 10 & 3 & 10 \\
\hline casado & 10 & 33,3 & 3 & 10 & 13 & 43,3 \\
\hline unido & 0 & 0 & 1 & 3,3 & 1 & 3,3 \\
\hline Separado & 1 & 3,3 & 2 & 6,7 & 3 & 10 \\
\hline Divorciado & 2 & 6,7 & 2 & 6,7 & 4 & 13,3 \\
\hline Viudo & 1 & 3,3 & 5 & 16,7 & 6 & 20 \\
\hline total & 14 & 46,6 & 16 & 53,4 & 30 & 100 \\
\hline
\end{tabular}


En la tabla 2, las personas de la tercera edad participan en la investigación el 73,3 \% convive con un familiar o su pareja con buenas relaciones y el $26,7 \%$ viven solos los mismos que mantienen contacto con sus familiares por medio de comunicación.

\section{Tabla 2.}

Convivencia de los adultos mayores de la parroquia Antonio José Holguín en el 20192020.

\begin{tabular}{|l|c|c|}
\hline Convivencia & $\mathbf{n}$ & $\%$ \\
\hline Familiar & 22 & 73,4 \\
\hline No familiar & 1 & 3,3 \\
\hline Solo & 7 & 23,3 \\
\hline Total & 30 & 100 \\
\hline
\end{tabular}

\section{Bienestar físico, bienestar mental y bienestar social}

En la tabla 3, se evidencia que en las actividades básicas de la vida diaria el $90 \%$ de los adultos mayores son independientes, en cuando actividades instrumentales de la vida diaria el 63\% necesita cierta ayuda. En $100 \%$ no sugiere déficit cognitivo y no depresión que participan en la investigación. En cuanto al bienestar social es aceptado $67 \%$ por lo que cuentan con su familia. 


\section{Tabla 3.}

Escala Geriátrica de los adultos mayores de la parroquia de Antonio José Holguín en el 2019-2020.

\begin{tabular}{|c|c|}
\hline Escala geriátrica integral MSP & Porcentaje \\
\hline \multicolumn{2}{|c|}{ Actividades básicas de la vida Diaria: Índice de Katz } \\
\hline Independiente & 90 \\
\hline Dependencia leve & 7 \\
\hline Dependencia moderada & 3 \\
\hline \multicolumn{2}{|c|}{$\begin{array}{l}\text { Actividades instrumentales de la vida diaria. Escala de Lawton y } \\
\qquad \text { Brody modificada }\end{array}$} \\
\hline Hasta 8 puntos independiente & 37 \\
\hline De 8 a 20 puntos necesita cierta ayuda & 63 \\
\hline \multicolumn{2}{|c|}{ Evaluación cognitiva Mms modificada } \\
\hline Ente 14 y 19 no sugiere déficit cognitivo & 100 \\
\hline \multicolumn{2}{|c|}{ Escala de Depresión Yesavage Modificada } \\
\hline Normal (0-5) & 100 \\
\hline \multicolumn{2}{|c|}{ Escala de valoración social de Guijón } \\
\hline De 5 o meno bajo riesgo socia & 33 \\
\hline De 6-9 aceptable & 67 \\
\hline
\end{tabular}

Como se evidencia en la tabla 4, el medio más utilizado de comunicación en el $90 \%$ de los casos es la televisión, la radio en el $86,7 \%$ de los adultos mayores. Para comunicarse con sus familiares utiliza teléfono celular en el $50 \%$ con lo que mantienen buenas relaciones. 


\section{Tabla 1.}

Medios de Comunicación en los adultos mayores de la parroquia Antonio José Holguín en el 2019-2020.

\begin{tabular}{|l|c|c|}
\hline Tecnología de la comunicación & Frecuencia & Porcentaje \\
\hline Radio & 26 & 86,7 \\
\hline Televisión & 27 & 90 \\
\hline Teléfono convencional & 7 & 23,3 \\
\hline Teléfono Celular & 15 & 50 \\
\hline Computadora & 4 & 13,3 \\
\hline Internet & 6 & 20 \\
\hline Ninguno & 1 & 3,3 \\
\hline
\end{tabular}

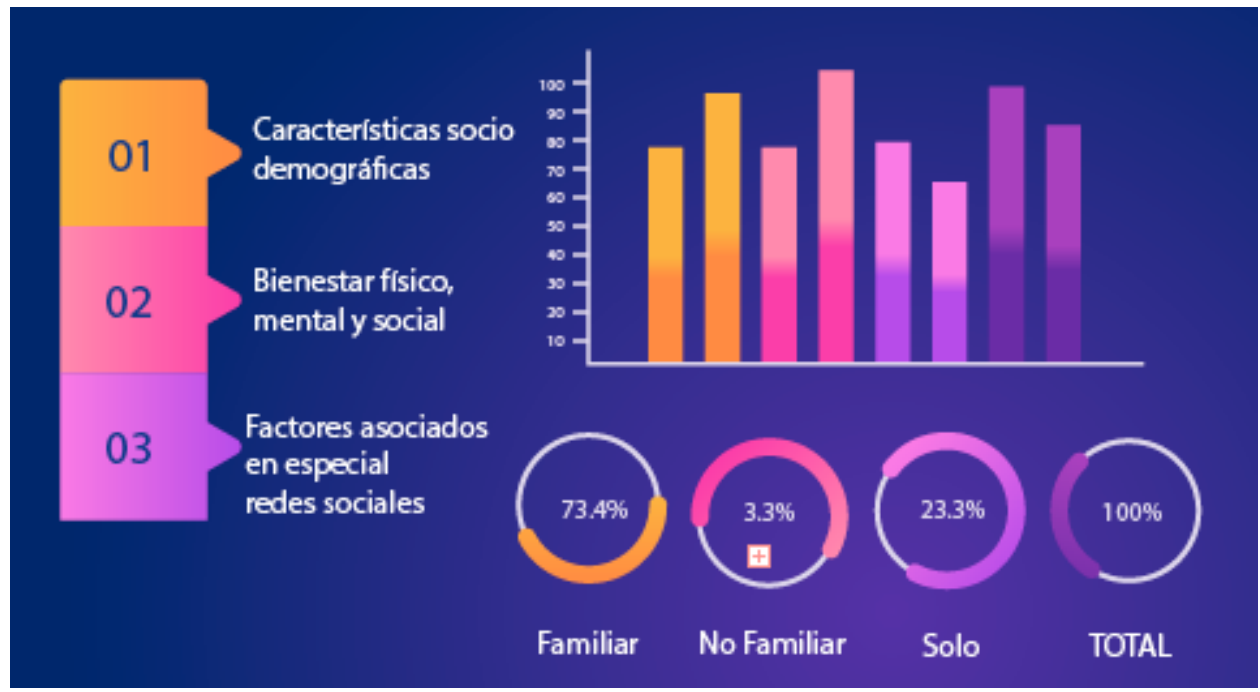

Figura 2. Resumen general de resultados obtenidos en la investigación. Fuente: Elaboración propia. 


\section{DISCUSIÓN}

En esta investigación se evidencia que el uso de medios de comunicación genera un envejecimiento activo porque mejora el bienestar físico, mental y social. El teléfono celular es utilizado con el fin de tener comunicación con familia y amigos. Para tener información los más utilizados son televisión y la radio.

Los efectos del uso de las redes sociales es mejorar su autoeficacia y reducir su soledad. Este apoyo social de compañeros o familiares influye en su motivación y comportamiento y su vida cotidiana ${ }^{10}$. Los hombres mayores tienen mayor satisfacción con la vida que las mujeres con el uso de redes sociales. Las estrategias de promoción de uso de las mismas deben ser por grupo de edad o nivel de educación para una buena adherencia 11.

El uso social de las redes predice un mayor bienestar por tener un papel en la actividad social en línea. Esto ayuda al adulto mayor un sentido de pertenencia y recibe su apoyo social, además el participar en diversas actividades ${ }^{12}$. El uso de Facebook para la creación de grupos de apoyo, actividades comunitarias con un impacto en la participación en la misma ${ }^{13}$. Además, las personas adultas mayores tienen preocupación por las relaciones con la familia, fiabilidad y calidad de los medios ocupadas ${ }^{14}$.

El uso de tecnología en adultos mayores revela el apego emocional que fomenta comunicarse con amigos, familiares y vecinos de la comunidad ${ }^{15}$. Además, una relación positiva entre la frecuencia con el uso y sus percepciones de autoestima y va aliviar la soledad ${ }^{16}$. El uso de teléfonos inteligentes es el más utilizado por los adultos mayores porque da un sentido de pertenencia, confianza, un bienestar psicológico ${ }^{17}$. Lo que genera autoeficacia, autocuidado, empoderamiento y además un buen desempeño de las personas de la tercera edad ${ }^{18}$. 


\section{CONCLUSIONES}

Los adultos mayores al tener medios de comunicación le permiten mantenerse informado y un permanente contacto con sus familiares y amigos. Se evidencia que la comunicación mejora el bienestar físico, mental y social, lo que conservan de manera independiente y evita la depresión.

El envejecimiento activo es poder realizar las actividades del diario vivir y tomar las decisiones de su propia vida de una manera independiente. Al tener un nivel cognitivo normal a través de mantenerse parte su familia y comunidad con la ayuda de los medios de comunicación.

\section{CONFLICTO DE INTERÉS}

Los autores declaran que no tienen conflicto de interés en la publicación de este artículo.

\section{FINANCIAMIENTO}

Autofinanciado

\section{AGRADECIMIENTO}

Al Centro de Salud Tipo A Antonio José Holguin. Centro de posgrado de la Universidad Técnica de Ambato. Especialización en Medicina Familiar y Comunitaria, segunda cohorte; por el apoyo en el desarrollo de la investigación. 
Victoria Noemi Gutiérrez-Martínez; Patricio Rodrigo-Maldonado; Mayra Alexandra Vela-Chasiluisa

\section{REFERENCIAS}

1. Um, J, Zaidi, A, Choi, S-J. Active Ageing Index in Korea - Comparison with China and EU countries. Asian Soc Work Pol Rev. 2019; 13: 87- 99. https://doi.org/10.1111/aswp.12159

2. Lazo-Porras $M$, Ortiz-Soriano $V$, Moscoso-Porras $M$, Runzer-Colmenares FM, Málaga G, Jaime Miranda J. Cognitive impairment and hypertension in older adults living in extreme poverty: a cross-sectional study in Peru. BMC Geriatr. 2017 Oct 26;17(1):250. doi: 10.1186/s12877-017-0628-8. PMID: 29073885; PMCID: PMC5659043.

3. Perseguino M, de-Moraes-Horta, A, Ribeiro-Circéa A. The family in face of the elderly's reality of living alone. Rev. Bras. Enferm. [Internet]. 2017; 70(2): 235241.

4. Machado J. Ecuador tendrá 1,3 millones de adultos mayores a finales de 2020 . Primicias [Ecuador will have 1.3 million older adults by the end of 2020]. [Internet]. 2019. https://n9.cl/dudb

5. Griol, D, Molina, J M, Sanchis, A. A multimodal conversational coach for active ageing based on sentient computing and $\mathrm{m}$-health. Expert Systems. 2020; 37:e12454. https://doi.org/10.1111/exsy.12454

6. Mansvelt, J., Elms, J., and Dodds, S. (2020) Connecting meanings of ageing, consumption, and information and communication technologies through practice. Geographical Research, 58: 289-299. https://doi.org/10.1111/1745$\underline{5871.12392 .}$

7. Baker S, Warburton J, Waycott J, Batchelor F, Hoang T, Dow B, Ozanne E, Vetere F. Combatting social isolation and increasing social participation of older adults through the use of technology: A systematic review of existing evidence. Australas J Ageing. 2018 Sep; 37(3):184-193. doi: 10.1111/ajag.12572. Epub 2018 Jul 19. PMID: 30022583 
Victoria Noemi Gutiérrez-Martínez; Patricio Rodrigo-Maldonado; Mayra Alexandra Vela-Chasiluisa

8. Casado Muñoz R, Lezcano Barbero F. Inclusión digital y envejecimiento activo: la participación de los mayores en las redes sociales [Digital inclusion and active aging: the participation of the elderly in social networks]. AULA_ABIERTA [Internet]. 31 de enero de 2018; 47(1):113-22. https://doi.org/10.17811/rifie.47.1.2018.113-122

9. Fuss BG, Dorstyn D, Ward L. Computer-mediated communication and social support among community-dwelling older adults: A systematic review of crosssectional data. Australas J Ageing. 2019 Dec;38(4):e103-e113. doi: 10.1111/ajag.12703. Epub 2019 Aug 4. PMID: 31379102.

10. Rivoir, A., Morales, M., \& Casamayou, A. Usos y percepciones de las tecnologías digitales en personas mayores. Limitaciones y beneficios para su calidad de vida. Revista Austral de Ciencias Sociales, (2019); (36), 295-313. https://doi.org/10.4206/rev.austral.cienc.soc.2019.n36-15

11.Zhou B, Liu X, Yu P. Toward successful aging: The Chinese Health Criteria for the Elderly. Aging Med (Milton). 2018 Jul 6;1(2):154-157. doi: 10.1002/agm2.12028. PMID: 31942493; PMCID: PMC6880742.

12. Szabo A, Allen J, Stephens C, Alpass F. Longitudinal Analysis of the Relationship Between Purposes of Internet Use and Well-being Among Older Adults. Gerontologist. 2019 Jan 9;59(1):58-68. doi: 10.1093/geront/gny036. PMID: 29688332.

13. Rebecca Ping Yu, Nicole B. Ellison \& Cliff Lampe. Facebook Use and Its Role in Shaping Access to Social Benefits among Older Adults, Journal of Broadcasting \& Electronic Media, (2018); 62:1, 71-90, DOI: 10.1080/08838151.2017.1402905

14. Soja, E. Information and Communication Technology in Active and Healthy Ageing: Exploring Risks from Multi-generation Perspective, Information Systems Management, (2017); 34:4, 320-332, DOI: $\underline{\text { 10.1080/10580530.2017.1366217 }}$

15. Padial Espinosa M, Pinzón Pulido, S, Espinosa Almendro J, Kalache A, Garrido Peña F. Longevidad y revolución digital. Contribución de las tecnologías de la información y de la comunicación al envejecimiento saludable [Longevity and digital revolution. The contribution of information and communication technologies to healthy aging]. Gerokomos [Internet]. 2020; 31(1): 6-14. 
Victoria Noemi Gutiérrez-Martínez; Patricio Rodrigo-Maldonado; Mayra Alexandra Vela-Chasiluisa

16. Wilson C. Is it love or loneliness? Exploring the impact of everyday digital technology use on the wellbeing of older adults. Ageing and Society. Cambridge University Press; 2018;38(7):1307-31.

17. Muñoz Alarcón MT, Sáez Carreras J, Campillo Díaz M. Las TIC y los procesos de envejecimiento activo: ¿una promesa incumplida?. MÁRGENES [Internet]. 2020; 1(2):34-47.

18. Brito Tábatta Renata Pereira de, Nunes Daniella Pires, Duarte Yeda Aparecida de Oliveira, Lebrão Maria Lúcia. Redes sociais e funcionalidade em pessoas idosas: evidências do estudo Saúde, Bem-Estar e Envelhecimento (SABE). Rev. bras. epidemiol. [Internet]. 2018; 21 (Suppl 2): e180003.

(C2020 por los autores. Este artículo es de acceso abierto y distribuido según los términos y condiciones de la licencia Creative Commons Atribución-NoComercial-Compartirlgual 4.0 Internacional (CC BY-NC-SA 4.0) (https://creativecommons.org/licenses/by-nc-sa/4.0/). 